\title{
Creating High-Harmonic Beams with Controlled Orbital Angular Momentum
}

\author{
Genevieve Gariepy, ${ }^{1,}{ }^{*}$ Jonathan Leach, ${ }^{2,3}$ Kyung Taec Kim, ${ }^{1,4,5}$ T. J. Hammond, ${ }^{1}$ \\ E. Frumker, ${ }^{1,6}$ Robert W. Boyd, ${ }^{2,7}$ and P. B. Corkum ${ }^{1, \dagger}$ \\ ${ }^{1}$ Joint Attosecond Science Laboratory, University of Ottawa and National Research Council, Ottawa K1A OR6, Canada \\ ${ }^{2}$ Department of Physics, University of Ottawa, Ottawa KIN 6N5, Canada \\ ${ }_{3}^{3} I P a Q S$, SUPA, Heriot-Watt, Edinburgh EH14 4AS, United Kingdom \\ ${ }^{4}$ Center for Relativistic Laser Science, Institute for Basic Science (IBS), Gwangju 500-712, Republic of Korea \\ ${ }^{5}$ Department of Physics and Photon Science, Gwangju Institute of Science and Technology (GIST), \\ Gwangju 500-712, Republic of Korea \\ ${ }^{6}$ Department of Physics, Ben-Gurion University, Beer-Sheva 84105, Israel \\ ${ }^{7}$ Institute of Optics, University of Rochester, Rochester, New York 14627, USA
}

(Received 19 December 2013; published 7 October 2014)

\begin{abstract}
A beam with an angular-dependant phase $\Phi=\ell \phi$ about the beam axis carries an orbital angular momentum of $\ell \hbar$ per photon. Such beams are exploited to provide superresolution in microscopy. Creating extreme ultraviolet or soft-x-ray beams with controllable orbital angular momentum is a critical step towards extending superresolution to much higher spatial resolution. We show that orbital angular momentum is conserved during high-harmonic generation. Experimentally, we use a fundamental beam with $|\ell|=1$ and interferometrically determine that the harmonics each have orbital angular momentum equal to their harmonic number. Theoretically, we show how any small value of orbital angular momentum can be coupled to any harmonic in a controlled manner. Our results open a route to microscopy on the molecular, or even submolecular, scale.
\end{abstract}

DOI: 10.1103/PhysRevLett.113.153901

PACS numbers: 42.65.Ky, 42.50.Tx

Light beams carry orbital angular momentum (OAM) when their Poynting vector spirals around the propagation direction. Allen et al. showed that Laguerre-Gaussian modes have such a property [1]. These modes are described by an angular-dependant phase $\Phi=\ell \phi$, where the topological charge $\ell$ is an index that indicates the OAM carried by each photon in the beam and $\phi$ is the azimuthal angle. Laguerre-Gaussian modes do not have a well-defined phase at their center, which leads to their characteristic zerointensity center. These two characteristics, the spiraling Poynting vector and the zero on-axis intensity, have given rise to a rich variety of applications: in optical communications, Laguerre-Gaussian beams are used to encode multiple bits in one photon [2,3]; in biophotonics, Stimulated Emission Depletion (STED) microscopy exploits the zero intensity to achieve resolution up to $\lambda / 25$ [4-7]; in optical manipulation, light beams are used to trap small objects or molecules and OAM is transferred to induce rotation in the trapped objects $[8,9]$.

Previous experiments have shown that OAM can be transferred to shorter wavelengths by perturbative nonlinear processes [10-12]. However, high-harmonic generation is not perturbative [13]: the total number of photons involved in a high-order process is not well defined. To date, the only experiment to consider coupling OAM from a fundamental to the $n$th harmonic demonstrated that the topological charge of all high-order harmonics is equal to that of the fundamental [14]. In contrast, the only theoretical treatment predicts it should be equal to the harmonic number [15].
We develop a model based on the strong-field approximation and confirm that orbital angular momentum is conserved in high-harmonic generation. Experimentally, using argon as the nonlinear medium and an $800 \mathrm{~nm}$ pump beam with $\ell= \pm 1$, we measure that the 11 th, 13th, and 15th harmonics have topological charges of $\ell=11$, $\ell=13$, and $\ell=15$, respectively. Thus, both our model and experimental results confirm that the topological charge of the $n$th harmonic is equal to $n$ times that of the fundamental; i.e., $\ell_{\text {harmonic }}=n \ell_{\text {fundamental }}$. However, this transfer is very sensitive to any imperfections in the fundamental beam. Therefore, we introduce a robust new method where any value of OAM can be transferred to any high-order harmonic using nonlinear wave mixing. Thus, we open a new wavelength range for OAM studies and a new direction for high-harmonic spectroscopy.

Our model treats the interaction of the pump beam with atoms having characteristics of argon [16]. We choose parameters to represent our experimental configuration, which are $\ell=1, \lambda=800 \mathrm{~nm}$, and a pulse duration of $30 \mathrm{fs}$. We represent the complex amplitude of the pump as a two-dimensional matrix, and at each point we calculate the single-atom response using the strong-field approximation (see Supplemental Material, Sec. S1 [17]) [18,19]. From this model, we obtain the time-dependant electric field of the attosecond pulse train generated at each transverse point of the input beam. The amplitude and phase of each harmonic in the far field of the gas jet is obtained by performing a Fourier transform in time and in space. 
The intensity profile that we predict for each harmonic is characteristic of an optical vortex. The model also predicts that the phase of the $n$th harmonic is equal to the phase of the fundamental multiplied by $n$. In other words, our model predicts conservation of OAM in high-harmonic generation, which is a natural extension to what has already been reported for second-harmonic generation, and in agreement with a recent theoretical analysis [15].

Experimentally, we generate high-order harmonics by focusing a $\lambda=800 \mathrm{~nm}$ pump laser beam into a gas jet of argon with a $30 \mathrm{~cm}$ lens; see Fig. 1 . The generated harmonics propagate to the far field and are detected by an imaging spectrometer. The spectrometer is composed of a vertical slit, a 1200 lines/mm grating, a microchannel plate (MCP), and a CCD camera; the plane of the slit is imaged onto the MCP using the imaging grating (Hitachi 001-0266), and the CCD camera records the intensity on the phosphor screen of the MCP. The grating separates the harmonic orders by mapping wavelength to the horizontal axis of the MCP. In order to measure the full transverse profile of each harmonic, the slit and grating are both moved by a microstage. This setup allows us to measure the transverse intensity profiles from the 11th to the 15th harmonic. To impart OAM to the pump beam, we place a spatial light modulator (SLM) before the focusing lens. We use a $100 \mathrm{~Hz}$ Ti:sapphire laser of $1 \mathrm{~mJ}$ per pulse incident on the SLM (PLUTO-BB-HR from Holoeye), which has a low retardance panel for increased phase stability. A forked diffraction pattern is displayed on the SLM [20], creating an $\ell=1$ beam in the first diffraction order. The grating efficiency is approximately $78 \%$. A long period-grating pattern is used to minimize angular dispersion in the first diffraction order (see Sec. S2 of the Supplemental Material [17]) [21,22]. The separation between the zeroth and the first order at the focus is $121 \mu \mathrm{m}$, around 3.5 times the beam waist diameter at focus.
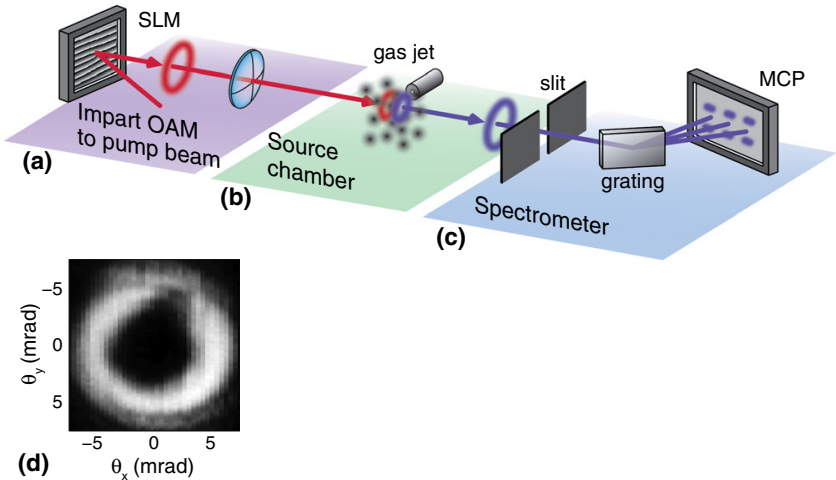

FIG. 1 (color online). (a) OAM is imparted to the pump with a spatial light modulator. (b) High-order harmonics are generated in argon and (c) measured in a spectrometer. The grating allows us to resolve each harmonic. Moving the slit and grating horizontally allows us to recover the full transverse profile of the harmonics. (d) Intensity profile for the 13th harmonic. All harmonics show the same characteristic intensity profile.
For every harmonic order, we observe a doughnut-shaped mode with an intensity zero on the beam axis (see Fig. 1 for the 13th harmonic). Thus, the intensity profiles of the experimentally produced harmonics correspond to the theoretical prediction of the generation of optical vortices.

To assess the OAM content of the mode, we must measure the phase structure of the beam. A measurement can be achieved by interference with a flat-phase reference. In the laboratory, the interference between a beam with a topological charge $\ell$ and an inclined Gaussian beam satisfies this condition. It produces an $\ell$-forked fringe pattern [20]; the difference in the number of fringes on either side of the interference pattern is the charge of the optical vortex. To create a reference beam in the extreme ultraviolet, the diffraction grating displayed on the SLM is modified to decrease its diffraction efficiency. We control the relative powers in the zeroth and first diffraction orders by modifying the phase depth of the blazed diffraction grating. A full $2 \pi$ modulation maximizes the power in the first diffracted order; reducing the modulation depth reduces the power in the first order and increases the power in the zeroth order. It follows that harmonics can then be generated from both the zeroth and first order. The zeroth order contains no OAM and consequently generates Gaussian harmonics that we use as references (Fig. 2). The modeled outcome of such an experiment, including space-time coupling induced by the SLM grating, is shown in Fig. 2 and is consistent with the conservation of OAM in the generation of high harmonics; i.e., $\ell_{\text {harmonic }}=$ $n \ell_{\text {fundamental }}$. The difference of fringes between the rightand left-hand sides of the interference pattern is the topological charge $\ell_{\text {harmonic }}=n$ (see upper left-hand images of Fig. 2). Additionally, a vertical Fourier transform allows us to conveniently evaluate the OAM of the generated mode: the number of fringes along the horizontal axis is equal to $\ell+1$ (see lower left-hand images of Fig. 2).

With the reference beam, we measure the OAM content for the harmonics $n=11,13$, and 15 produced from a $\ell= \pm 1$ pump beam (Fig. 2). For the 11th harmonic, we measure a difference of 11 fringes between the left- and right-hand sides of the interference pattern and 12 lobes in the vertical Fourier transform (see Sec. S3 of the Supplemental Material for details [17]). The patterns are reversed in the right-hand images where the charge of the pump beam is $\ell=-1$. Thus, both our model and our experiment confirm that the 11th harmonic contains 11 times the OAM of the pump.

Because of the increasing spatial frequency, the decreasing overlap between the OAM and reference beam, and the lower divergence of harmonics 13 and 15, the fringe visibilities decrease for the harmonics $n=13$ and 15 . We thus use a different method to analyze the patterns: we estimate the number of fringes by measuring the size of the beam and the fringe frequency on both sides. In Fig. 3, we approximate where there is a fringe on the top and bottom 
(a)
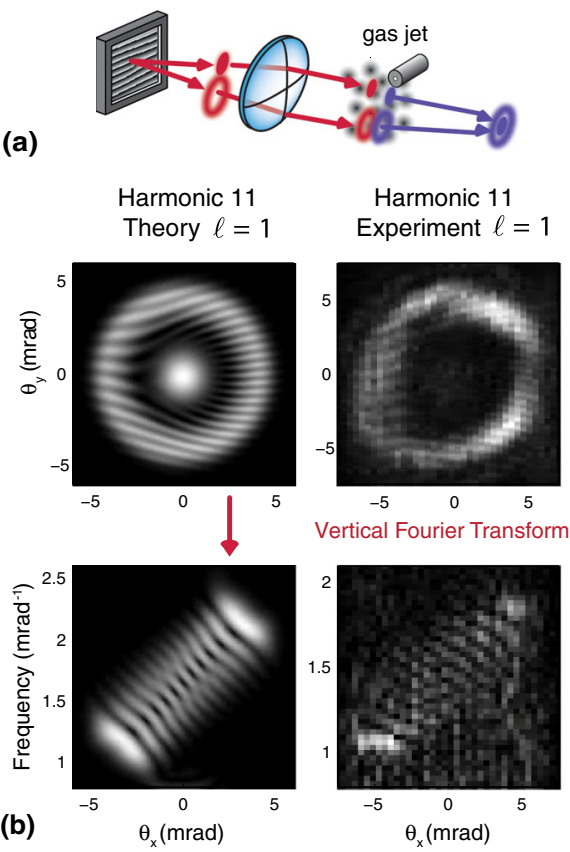

Harmonic 11 Experiment $\ell=-1$
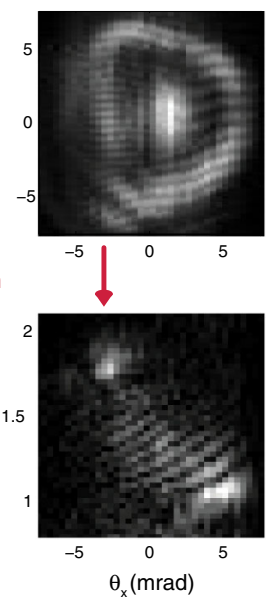

FIG. 2 (color online). (a) Generating harmonics from two distinct beams at focus creates an OAM beam and a Gaussian reference beam. (b) The interference, in the far field, of the harmonic beam containing OAM and a Gaussian harmonic beam results in a $n$-fork interference pattern as shown in the upper lefthand image, where $n$ is the OAM per photon of the $n$th harmonic. The vertical Fourier transform of such a pattern exhibits increasing frequency from one side of the beam to the other, with $n+1$ steps, as shown in the lower left-hand image. Experimentally, the interference patterns and their Fourier analysis are similar to those predicted. For harmonic 11, we count a difference of 11 fringes between left and right for harmonics produced from a $\ell= \pm 1$ fundamental beam. The charge $\ell$ of the harmonic is reversed when we inverse the charge of the fundamental beam. These results confirm that OAM is conserved in high-harmonic generation.

that is present on both sides of the pattern and trace its position by a line. We then use the Fourier analysis to have a value of the fringe frequency at two $\theta_{x}$ positions. We choose two positions where the frequency component is bright; at those two positions, we measure the size of the beam by taking the distance $\Delta \theta_{y}$ between the two red lines traced on the interference patterns. We find the number of fringes by multiplying the fringe frequency by the size of the beam. Using this procedure, we find a difference of $13.1 \pm 1.5$ and $14.9 \pm 1.5$ fringes for harmonics 13 and 15 , respectively. Again, we find that orbital angular momentum is conserved.

As we increase in harmonic number, the OAM transferred should continue to increase as $\ell_{\text {harmonic }}=$ $n \ell_{\text {fundamental }}$. However, beams with shorter wavelengths and higher OAM will be highly sensitive to aberrations and defects in the experimental setup. For example, the slight imperfection placed on the beam by the SLM will eventually impose a limit. To avoid problems that come with

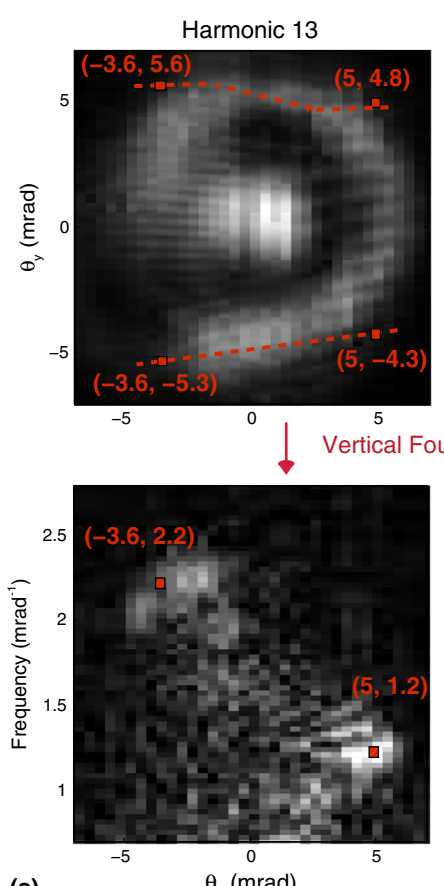

(a)

$\theta_{x}(\mathrm{mrad})$

(b)
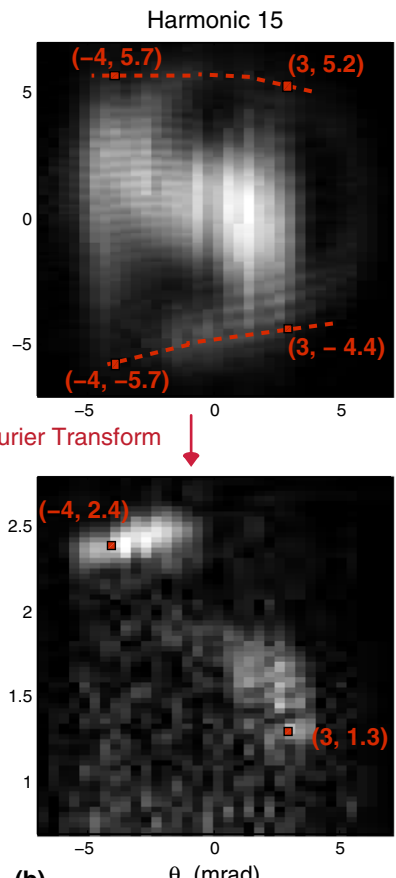

FIG. 3 (color online). In the two top figures, we approximately trace the fringes on top and bottom that are linked from left to right. We measure the beam size between these fringes at two $x$ positions (with uncertainty $\pm 0.1 \mathrm{mrad}$ ) and measure the frequency at these positions with the bottom figures (uncertainty $\left.\pm 0.1 \mathrm{mrad}^{-1}\right)$. (a) For harmonic 13: on the left $\left(\theta_{x}=\right.$ $-3.6 \mathrm{mrad})$, the beam size is $\Delta \theta_{y}=(5.6+5.3) 10.90 \pm$ $0.14 \mathrm{mrad}$; on the right $\left(\theta_{x}=5 \mathrm{mrad}\right)$, the size is $\Delta \theta_{y}=$ $(4.8+4.3) 9.10 \pm 0.14 \mathrm{mrad}$. The frequency on the left is $2.2 \mathrm{mrad}^{-1}$ so that the number of fringes is $23.98 \pm 1.13$. The frequency on the right is $1.2 \mathrm{mrad}^{-1}$ so that the number of fringes is $10.92 \pm 0.93$. The difference is $13.1 \pm 1.5$ fringes. (b) For harmonic 15: on the left $\left(\theta_{x}=-4 \mathrm{mrad}\right)$, the beam size is $\Delta \theta_{y}=(5.7+5.7) 11.40 \pm 0.14 \mathrm{mrad} ; \quad$ on the right $\left(\theta_{x}=\right.$ $3 \mathrm{mrad})$, the size is $\Delta \theta_{y}=(5.2+4.4) 9.60 \pm 0.14 \mathrm{mrad}$. The frequency on the left is $2.4 \mathrm{mrad}^{-1}$ so that the number of fringes is $27.36 \pm 1.19$. The frequency on the right is $1.3 \mathrm{mrad}^{-1}$ so that the number of fringes is $12.48 \pm 0.98$. The difference is $14.9 \pm 1.5$ fringes. In both cases, the results show that OAM is conserved.

increasing charge $\ell_{\text {harmonic }}$, we use our model to demonstrate how any small OAM value can ge given to any high harmonic. The core of our method is to generate harmonics from a forked-grating pattern inside the gas jet (see Fig. 4). To obtain a forked-grating interference pattern in the gas, we combine a main pump beam along the principal axis of propagation with a control beam that has $\ell=1$ (or more) arriving at a small incident angle [23]. This interference pattern is analogous to the forked pattern displayed on a spatial light modulator to generate OAM beams.

For the simulation we use an $800 \mathrm{~nm}$ Gaussian beam propagating forward with an intensity of $10^{14} \mathrm{~W} / \mathrm{cm}^{2}$ at focus, and a $400 \mathrm{~nm}, \ell=1$ beam arriving at an angle of $2.3^{\circ}$ with $10 \%$ of the main beam intensity and $50 \%$ of the main beam size at the SLM. At focus, the perturbing beam 

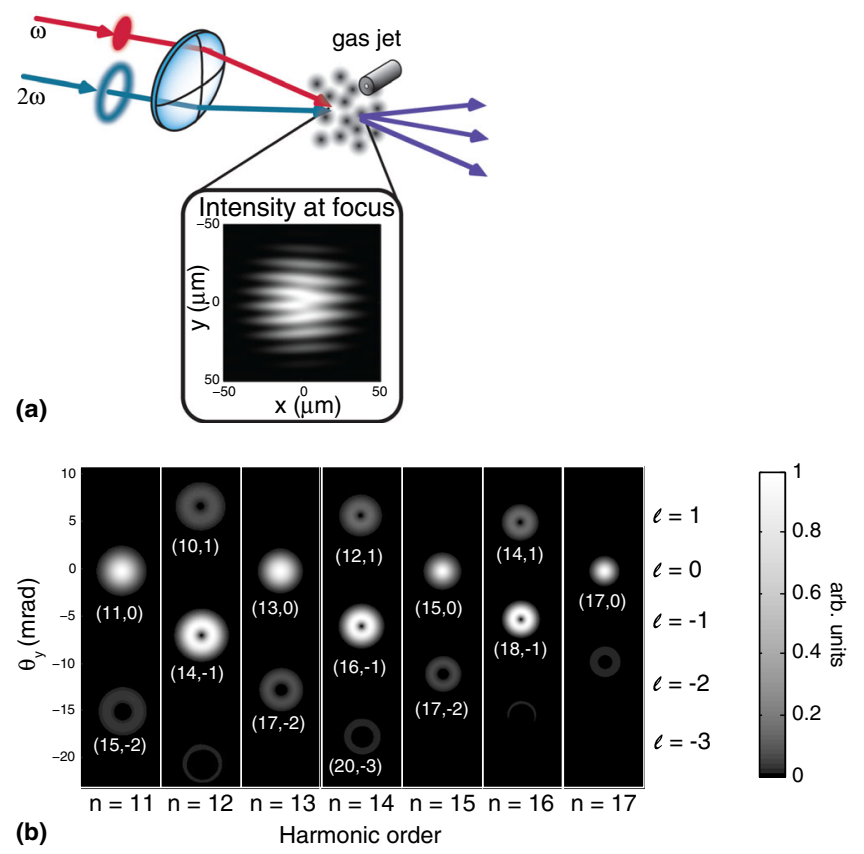

FIG. 4 (color online). (a) Controlling high-harmonic generation with a second-harmonic, $\ell=1$ beam incident at an angle creates a rapidly moving grating at the focal plane, shown here at a given instant. (b) Harmonics are created in the different diffraction orders, from a combination of $\left(m_{\omega}, m_{2 \omega}\right)$ photons, where $m_{\omega}$ is the number of fundamental photons and $m_{2 \omega}$ is the number of control photons involved in the process. The position at the far field is determined by conservation of linear momentum, and the orbital angular momentum of each harmonic beam is given by $m_{2 \omega}$ times the OAM of the control beam.

has $2.6 \%$ of the main beam's peak intensity. These parameters are similar to those used in a recent experiment studying how high-harmonic generation can be perturbed [23]. Using a control beam that is the second harmonic of the pump beam is not essential, but it is convenient because we can identify the net number of control beam photons contributing to a harmonic by both its direction and its harmonic order. Figure 4 shows the far field of the harmonics, where a range of OAM values is generated in the different diffraction orders. Although high-harmonic generation is a nonperturbative process, it is perturbative with respect to the control beam [23]. In Fig. 4, the Gaussianshaped harmonics on axis $\left(\theta_{y}=0 \mathrm{mrad}\right)$ are harmonics of the main $800 \mathrm{~nm}$ beam. They have no OAM. The harmonics above and below $\left(\theta_{y} \approx 7 \mathrm{mrad}\right)$ have one $400 \mathrm{~nm}$ photon mixed with $n \pm 2800 \mathrm{~nm}$ photons. All of these harmonics have the intensity (shown) and phase (not shown) structure of a $\ell= \pm 1$ charged beam. Higher-order perturbative peaks are seen at $\theta_{y} \approx 15 \mathrm{mrad}$ and $\theta_{y} \approx 22 \mathrm{mrad}$. They have OAM of $\ell=-2$ and $\ell=-3$, respectively. As the harmonic generation is perturbative with respect to the control beam, the diffracted intensity will increase with the second-harmonic intensity and higher diffractive orders will appear and gain strength [23].
Before concluding we comment on the sensitivity of our results to beam imperfections. A Gaussian fundamental beam, interacting with an atomic medium under conditions where phase matching is maintained, produces high harmonics that are beautifully Gaussian in intensity and parabolic in phase [24]. This is because, in the far field of the fundamental (near where the jet is placed), there is a well-formed intensity maximum. Imperfections on the fundamental beam create spatial wings on the beam, but their influence on the high harmonics is strongly suppressed by the highly nonlinear nature of the process. In contrast, an OAM beam has a ring-shaped intensity distribution at the focus and small imperfections to the ring are "amplified" by the high-order nonlinearity. This influences both the phase and the amplitude structure of the produced harmonics. Coupling OAM onto a Gaussian fundamental using a perturbing beam gives us many of the advantages of Gaussian harmonic generation. We do not expect extreme sensitivity to small imperfections on the perturbing beam with this approach, and we expect it will be effective for producing harmonics.

Controlling the OAM transfer to high-order harmonics provides an accessible, tabletop source of high photon energy OAM beams that can be extended to $1.6 \mathrm{keV}$ [25]. X-ray focusing optics, such as zone plates, now allow soft $\mathrm{x}$ ray with a focal spot as small as $15 \mathrm{~nm}$, for photon energy between 250 and $1.8 \mathrm{keV}$ [26]. Therefore, focusing OAM beams to this scale seems feasible. In this spectral region, conversion efficiencies to a single harmonic approach $10^{-7}$ and megawatt power pulses could be produced. Not only do soft $\mathrm{x}$ rays promise enhanced resolution, but they have the added advantage of sensitivity to the environment near specific atomic species within molecules or solids. They will open nondipole spectroscopy for study [27].

A potential approach to STED-like microscopy is also opened. The critical idea in STED microscopy is to identify those molecules that were uninfluenced by an OAM beam. $\mathrm{X}$ rays offer at least one route to this end that was not available in the visible. We could employ an OAM soft$\mathrm{x}$-ray beam that is tuned to a specific core-to-valence transition. Excitation (or ionization) caused by this beam will shift all energy levels of the molecule. Now, for superresolution we must identify the unexcited molecules. We can achieve this using a Gaussian, resonant soft-x-ray beam tuned to a transition in the unionized molecule. Finally, Auger electrons from the decay of the excitation created by the Gaussian pulse can play the role of fluorescence photons in STED.

The authors have benefited from the help and advice of Dr. Andrei Naumov, Dr. David Villeneuve, and Prof. Daniele Faccio. Funding from the National Research Council of Canada, Natural Science and Engineering Research Council of Canada, and the US Air Force office of Scientific Research is also gratefully acknowledged. 
G. G. acknowledges the financial support of the National Science and Engineering Research Council of Canada, Grant No. 409519, and of the Fonds de recherche du Quebec-Nature et technologies, Grant No. 147901.

"Corresponding author. genevieve2.gariepy@gmail.com

Corresponding author. pcorkum@uottawa.ca

[1] L. Allen, M. W. Beijersbergen, R. J. C. Spreeuw, and J. P. Woerdman, Phys. Rev. A 45, 8185 (1992).

[2] J. Wang, J.-Y. Yang, I. M. Fazal, N. Ahmed, Y. Yan, H. Huang, Y. Ren, Y. Yue, S. Dolinar, M. Tur et al., Nat. Photonics 6, 488 (2012).

[3] G. Gibson, J. Courtial, M. J. Padgett, M. Vasnetsov, V. Pas'ko, S. M. Barnett, and S. Franke-Arnold, Opt. Express 12, 5448 (2004).

[4] K. I. Willig, S. O. Rizzoli, V. Westphal, R. Jahn, and S. W. Hell, Nature (London) 440, 935 (2006).

[5] S. W. Hell and J. Wichmann, Opt. Lett. 19, 780 (1994).

[6] S. W. Hell, Nat. Biotechnol. 21, 1347 (2003).

[7] V. Westphal, L. Kastrup, and S. Hell, Appl. Phys. B 77, 377 (2003).

[8] H. He, M. E. J. Friese, N. R. Heckenberg, and H. RubinszteinDunlop, Phys. Rev. Lett. 75, 826 (1995).

[9] A. T. O'Neil, I. MacVicar, L. Allen, and M. J. Padgett, Phys. Rev. Lett. 88, 053601 (2002).

[10] K. Dholakia, N. B. Simpson, M. J. Padgett, and L. Allen, Phys. Rev. A 54, R3742 (1996).

[11] J. Courtial, K. Dholakia, L. Allen, and M. J. Padgett, Phys. Rev. A 56, 4193 (1997).

[12] J. Strohaber, M. Zhi, A. V. Sokolov, A. A. Kolomenskii, G. G. Paulus, and H. A. Schuessler, Opt. Lett. 37, 3411 (2012).
[13] P. B. Corkum, Phys. Rev. Lett. 71, 1994 (1993).

[14] M. Zurch, C. Kern, P. Hansinger, A. Dreischuh, and C. Spielmann, Nat. Phys. 8, 743 (2012).

[15] C. Hernández-García, A. Picón, J. San Román, and L. Plaja, Phys. Rev. Lett. 111, 083602 (2013).

[16] V. S. Yakovlev, M. Ivanov, and F. Krausz, Opt. Express 15, 15351 (2007).

[17] See Supplemental Material at http://link.aps.org/ supplemental/10.1103/PhysRevLett.113.153901 for details on the theoretical model, the influence of space-time coupling on the experiment and the analysis of interference patterns.

[18] M. Lewenstein, P. Balcou, M. Y. Ivanov, A. L'Huillier, and P. B. Corkum, Phys. Rev. A 49, 2117 (1994).

[19] G. L. Yudin and M. Y. Ivanov, Phys. Rev. A 64, 013409 (2001).

[20] V. Y. Bazhenov, M. Vasnetsov, and M. Soskin, JETP Lett. 52, 429 (1990).

[21] H. Vincenti and F. Quéré, Phys. Rev. Lett. 108, 113904 (2012)

[22] K. T. Kim, C. Zhang, T. Ruchon, J.-F. Hergott, T. Auguste, D. Villeneuve, P. Corkum, and F. Quere, Nat. Photonics 7, 651 (2013).

[23] J. B. Bertrand, H. J. Wörner, H.-C. Bandulet, E. Bisson, M. Spanner, J.-C. Kieffer, D. M. Villeneuve, and P. B. Corkum, Phys. Rev. Lett. 106, 023001 (2011).

[24] E. Frumker, G. G. Paulus, H. Niikura, D. M. Villeneuve, and P. B. Corkum, Opt. Lett. 34, 3026 (2009).

[25] T. Popmintchev, M.-C. Chen, D. Popmintchev, P. Arpin, S. Brown, S. Aliauskas, G. Andriukaitis, T. Balciunas, O. D. Mcke, A. Pugzlys et al., Science 336, 1287 (2012).

[26] W. Chao, B. D. Harteneck, J. A. Liddle, E. H. Anderson, and D. T. Attwood, Nature (London) 435, 1210 (2005).

[27] M. van Veenendaal and I. McNulty, Phys. Rev. Lett. 98, 157401 (2007). 